\title{
Therapeutic gases used in balneotherapy and rehabilitation medicine - scientific relevance in the last ten years $(2011$ - 2020) - Synthetic literature review
}

\author{
MUNTEANU Constantin ${ }^{1,2,3}$, DOGARU Gabriela ${ }^{1,5,6}$, ROTARIU Mariana ${ }^{1,3}$, ONOSE Gelu $^{2,4}$
}

Editor: Mihail HOTETEU, Romanian Association of Balneology, hoteteu@yahoo.com

Corresponding author: Constantin MUNTEANU, E-mail: office@bioclima.ro

1. Romanian Association of Balneology, Bucharest, Romania

2. Teaching Emergency Hospital "Bagdasar-Arseni", Bucharest, Romania

3. Faculty of Medical Bioengineering, University of Medicine and Pharmacy "Grigore T. Popa", Iaşi, Romania

4. Faculty of Medicine, Department of Physical and Rehabilitation Medicine, University of Medicine and Pharmacy "Carol Davila", Bucharest,

5. Faculty of Medicine, University of Medicine and Pharmacy "Iuliu Hațieganu", Cluj-Napoca, Romania

6. Clinical Rehabilitation Hospital Cluj-Napoca, Romania

\section{Abstract}

Background. The medical relevance of therapeutic gases can range from use in burns and stroke victims to hypoxia therapy in children. However, medical gases such as oxygen, hydrogen, helium and xenon have recently come under increased exploration for their potential therapeutic use on various brain disease states, including traumatic brain injuries, hypoxia-ischemia and, cerebral hemorrhages.

Objective. This synthetic review aims to rigorously select related articles and identify within their content the main possible uses of therapeutic gases and physiological mechanisms. The objective of this article is to present the various therapeutic mechanisms that have been proposed in the current literature and the medical relevance of various therapeutic gases used in balneotherapy or medical rehabilitation.

Methods. To elaborate our synthesis review, we have searched for relevant open access articles in 6 international databases: Cochrane ${ }^{1}$, Elsevier ${ }^{1}$, NCBI/PubMed ${ }^{1}, \mathrm{NCBI} / \mathrm{PMC}^{1}, \mathrm{PEDro}^{1}$, and ISI Web of Knowledge/Science ${ }^{1}$, published from January 2011 until December 2020. The contextually quested keywords combinations/ syntaxes used are specified on this page. The eligible articles were analyzed in detail regarding pathologies addressed by therapeutic gases. All articles with any design (reviews, randomized controlled trials, nonrandomized controlled trials, case-control studies, cross-sectional studies), if eligible according to the above-mentioned selection methodology, containing in the title or abstract the above-mentioned combinations, were included in the analysis. Articles were excluded in the second phase if they did not reach the relevance criterion.

Results. Our search identified, first, 225 articles. After eliminating the duplicates, remained $\mathbf{1 8 0}$ articles. In the second phase, we applied a relevance criterion. Although our team - including the authors of this paper - have quite long and consistent expertise in achieving systematic literature reviews, the marked heterogeneity of both the item composing the subject we have approached and inherently the wase of presentations in the respective quite vast and diverse domain of medical gases, prevented us to fulfil, at least regarding this initial work, a systematic literature review. Instead, we succeeded to make a hopefully interesting and valuable narrative synthetic-related literature review. At the end of the second phase, $\mathbf{6 3}$ articles passed the relevance criterion and were included in this synthetic review.

Conclusions. Therapeutic gases are not fully understood and used adequately for sanogenic or medical purposes. More research is needed to fully understand the mechanisms and importance of therapeutic gases. The link between balneotherapy and medical rehabilitation regarding the usage of therapeutical gases emphasises the unity for this medical speciality.

Keywords: "Therapeutic gas"/ "Oxygen therapy"/ "Carbon dioxide"/ "CO2 therapy"/ "Carbon monoxide"/ "Mofette"/ "Hydrogen Sulfide"/ "H2S"/ "Helium"/ "Xenon"/ "Ozone therapy"/ "Radon"/ "Hydrogen therapy"/ "Nitric oxide"/ "Heliox" AND "Rehabilitation".
Authors:

Prof. dr. Gelu Onose, Ph.D.

Senior Physician in Physical and Rehabilitation Medicine (PRM) and Gerontology \& Geriatrics (GG), Head of the $\mathrm{P}$ (neural-muscular)RM Discipline/ Clinic Division - the National Reference Centre for NeuroRehabilitation - and of its RDI Nucleus, and RDI (ex) Director, at the Teaching Emergency Hospital "Bagdasar-Arseni"

Lecturer dr. Constantin Munteanu, Ph.D. Priority research areas: Bioclimatology, Hydrothermotherapy, and Physiology, President of the Romanian Association of Balneology, Vice President of the Romanian Society of Physical Medicine, Rehabilitation and Balneoclimatology,

Assist. prof. dr. Gabriela Dogaru, $\mathrm{PhD}$, Priority research areas: balneoclimatology, including the study of carbonated mineral waters and mofettes, molecular and cellular medicine, e-health systems, neurological and cardiovascular rehabilitation, development of spa, and rehabilitation tourism

Assoc. prof. dr. Mariana Rotariu, Ph.D.

Psychomotricity, Kinesitherapy. Kinesiprophilaxy, Massage. Complementary techniques, Occupational therapy, Rehabilitation, and techniques of functional reeducation
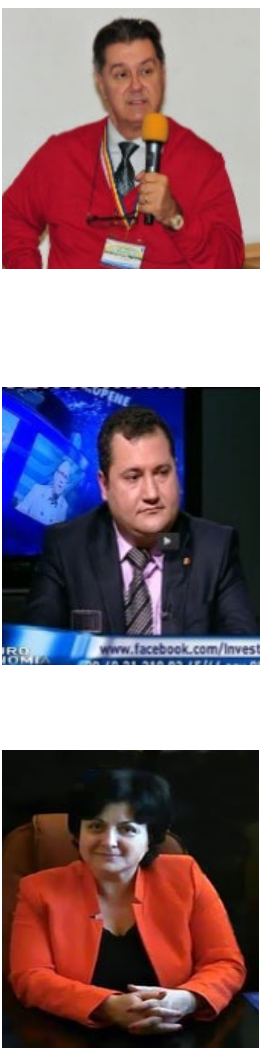

Reviewers:

Silișteanu Sînziana Călina, PhD

Stănescu Ioana, PhD

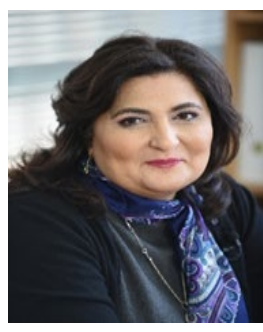




\section{INTRODUCTION}

One of the core elements of balneotherapy is the use of (natural) mineral waters, natural therapeutic gases, and peloids, often in health resorts (spas) (1). The net beneficial effect of balneotherapy is probably a result of mechanical, thermal, and chemical effects of balneary factors. For example, therapeutic activities of $\mathrm{CO} 2$ water baths rely on the synergism between hydrostatic pressure and the chemical properties of carbon dioxide, which acts directly on the blood vessels of the skin, causing vasodilation and increased oxygen use (2). For establishing Scientific Evidence in Thermal Medicine, we have to go beyond clinical trials. Due to a holistic perspective of balneotherapy, medical or sanaogenic use of natural mineral waters, steam, thermal gases, and peloids are not demanding sieve of a prospective, randomized, double-blind, longitudinal trial with a significance level of at least $95 \%$ (3).

On the other part, rehabilitation medicine is a larger umbrella, which also includes balneotherapy, with more therapeutical instruments, including the use of medicinal gases as part of a rehabilitation program.

Medical or therapeutic gases (3), seen as pharmaceutical molecules which play a certain role in our health, offer solutions to a wide array of medical needs, ranging from usual gases (oxygen and carbon dioxide), with known impact on our life, to gases like nitric oxide, carbon monoxide, and hydrogen sulfide -indicated to behave as messenger molecules in biological systems. Other gases, for example, helium and xenon, have even been shown to have neuroprotective effects following various brain injuries such as traumatic brain injury, acute ischemic stroke, perinatal hypoxia-ischemia, and cardiopulmonary bypass-induced neurologic and neurocognitive dysfunctions (4).

The medical relevance of therapeutic gases can range from burn and stroke victims to hypoxia therapy in children. However, medical gases such as oxygen, hydrogen, helium, and xenon have recently increased attention under their potential therapeutic use with various brain disease states, including traumatic brain injuries, hypoxia-ischemia, and cerebral hemorrhages. As the main objective, this article will review the multiple advances in therapeutical gases research and discuss the potential therapeutic applications and mechanisms concerning their use in the field of balneotherapy and medical rehabilitation. In balneotherapy, mofettic gas with a high concentration of carbon dioxide and hydrogen sulfide from natural sources are the natural factors taking, in this case, into consideration. For medical rehabilitation, as after a stroke, for example (5), supplementary oxygen, artificial $\mathrm{CO} 2$, ozone, helium, argon, xenon, nitric oxide, hydrogen can be discussed as therapeutic agents in different medical procedures.

\section{METHOD}

Literature Search Strategy. To elaborate our synthesis review, we have searched for relevant open access articles, in 6 international databases: Cochrane ${ }^{1}$, Elsevier ${ }^{2}$, NCBI/PubMed ${ }^{3}$, NCBI/PMC ${ }^{4}, \mathrm{PEDro}^{5}$, and ISI Web of Knowledge/Science ${ }^{6}$, published from January 2011 until December 2020. The contextually quested key words combinations/ syntaxes used in this respect were: "Therapeutic gas" + "Rehabilitation"

"Oxygen therapy" + "Rehabilitation"

"Carbon dioxide" + "Rehabilitation"

"CO2 therapy" + "Rehabilitation"

"Carbon monoxide" + "Rehabilitation"

"Mofette" + "Rehabilitation"

"Hydrogen Sulfide" + "Rehabilitation"

"H2S" + "Rehabilitation"

"Helium" + "Rehabilitation"

"Xenon" + "Rehabilitation"

"Ozone therapy" + "Rehabilitation"

"Radon" + "Rehabilitation"

"Hydrogen therapy" + "Rehabilitation"

"Nitric oxide" + "Rehabilitation"

"Heliox" + "Rehabilitation".

The eligible articles were analyzed in detail regarding pathologies addressed by therapeutic gases.

\section{Inclusion and Exclusion Criteria}

All articles with any design (reviews, randomized controlled trials, non-randomized controlled trials, casecontrol studies, cross-sectional studies), if eligible according to the above-mentioned selection methodology, containing in the title or abstract the abovementioned combinations, were included in the analysis.

Articles were excluded in the second phase if they did not reach the relevance criterion.

\section{RESULTS}

Our search identified, first, 225 articles. After eliminating the duplicates, remained $\mathbf{1 8 0}$ articles. In the second phase, we applied a relevance criterion. Although our team - including the authors of this paper - have quite long and consistent expertise in achieving systematic literature reviews (6), (7), (8), the marked heterogeneity of both the item composing the subject we have approached and inherently the wase of presentations in the respective quite vast and diverse domain of medical gases, prevented us to fulfil, at least regarding this initial work, a systematic literature review. Instead, we succeeded to make a hopefully interesting and useful narrative synthetic-related literature review. At the end of the second phase, $\mathbf{6 3}$ articles passed the relevance criterion and were included in this synthetic review.

\footnotetext{
1 https://www.cochrane.org/

${ }^{2}$ https://www.elsevier.com/

${ }^{3}$ https://pubmed.ncbi.nlm.nih.gov/

${ }^{4}$ https://www.ncbi.nlm.nih.gov/pmc/

${ }^{5}$ https://pedro.org.au/

${ }^{6}$ http://apps.webofknowledge.com/wOS GeneralSearch
} 


\section{Relevant data regarding natural therapeutic gases used in balneary resorts}

Balneotherapy has a long historical tradition for Europe and Asia. Romania can be the international bridge in this area due to its legendary practice, geographical position, and extraordinary natural resources. Each Balneary Resort begins its story by discovering natural factors whose therapeutical value has been clinically and experimentally proven by scientific personalities of that time (9). Physiotherapy is an important pillar of medical rehabilitation through the use of methods such as kinesiotherapy, balneotherapy, physical therapy, (in some cases) massage, and systematic exercise. Balneotherapy is beneficial for patients whose oxidative/antioxidative balance system is disturbed. Balneotherapy has long been used for treating obesity and its comorbidities. Balneotherapeutic sulfur baths have been shown to influence antioxidative status. The importance of antioxidant defense mechanisms in several degenerative diseases (atherosclerosis, diabetes, cataract, etc.) is largely explained (10) and is known as normalization effects conditioned by balneotherapy, including/ especially its way of intervention represented by oral ingestion (Crenotherapy, Inhalotherapy) (8).

Natural mofettes are gases resulting from natural postvolcanic emanations found in some regions of Romania, e.g. in the Harghita volcanic massif, from which carbon dioxide, present in a $95-98 \%$ proportion, is used as a therapeutic factor. Along with carbon dioxide, the mofettic gas contains small amounts of other volcanic emission gases: ammonium, sulfur, helium, and radon, which induce pulverization of $\mathrm{CO}_{2}$ molecules, increasing the penetration power of $\mathrm{CO}_{2}$. Mofettes also contain positive and negative air ions, 2,000-15,000 ions $/ \mathrm{cm} 3$, and have radioactivity of $0.3 \mu \mathrm{Ci} / 1$, without cancer risk, because radon has a concentration lower than $1 \%$. In Romania, there are natural mofettes in the spa resorts Covasna, Băile Tuşnad (11), Balvanyos, Malnaş, Harghita Băi, Buziaş, Borşa, Slănic Moldova, Sangeorz Băi. Artificial mofettes supplied from external sources developed in many medical rehabilitation centers. Mofettic gas is indicated for certain diseases, such as peripheral arterial disease stage 1,2 and 3, arteritis, venopathy, leg ulcers, chronic stable ischemic heart disease, stable hypertension stage 1 and 2 , dermatological, gynecological diseases, chronic degenerative rheumatism. However, the major indication of mofette is in secondary prevention of cardiovascular disease and cardiovascular disease rehabilitation. The exclusive use of the properties of carbon dioxide is possible by eliminating the mechanical factor, hydrostatic pressure, the thermal factor and gas bubble micromassage that are added to the carbonated mineral bath. The natural mofette is considered to induce an increase in blood flow through penetration of $\mathrm{CO} 2$ into the skin and muscles, which has a direct action on the smooth muscles of blood vessels, particularly on very small vessels and arterioles, an action estimated to be superior to that of peripheral vasodilatory drugs. Research performed on the natural mofette found in Covasna spa resort, Romania, showed some of its mechanisms of action, i.e. the effect of carbon dioxide inhaled by patients during mofette therapy (carbon dioxide concentration in the air 1.5-2 volumes $\%$, demonstrated by capnography); following inhalation of carbon dioxide, an increase in cerebral blood flow, up to $75 \%$, was found. During therapy, $\mathrm{CO} 2$ inhaled and dissolved in plasma, and absorbed into the skin, has many effects on the body; thus, at the level of peripheral circulation, it induces skin hyperemia by direct action on metarterioles, with an increase in skin temperature by $1.5-4$ degrees $\mathrm{C}$ and a $44 \%$ increase in skeletal muscle blood flow. There are also effects on cardiac hemodynamics by causing arterial vasodilation, a decrease in the pre-ejection period, an increase in the ejection period, and a reduction in blood pressure with consequences on peripheral oxygen use, which explains the improvement of cardiac performance parameters as well as the exercise capacity in coronary patients and patients with myocardial infarction sequelae (12).

The mofettes used for therapeutic purposes in Romania, in the Hospital of Rehabilitation in Cardiovascular diseases Covasna, are unique in the world. Here, carbon dioxide in the form of carbonaceous baths and mainly mofettes plays an essential role in primary prevention and secondary prevention and the rehabilitation of cardiovascular diseases. The types of cardiovascular disorders indicated for balneotherapy in Covasna for secondary prevention or rehabilitation are mainly represented by the following: obliterative arterial disease of the lower limbs, Burger thromboangiitis, chronic ischemic heart disease (all its forms), drug-controlled hypertension, heart valve disease without rhythm and hemodynamic disorders (including operated valvulopathies, after three months), post-stroke sequelae, chronic venous disease. In the literature, there are no data regarding the role of this unique therapy in the secondary prevention and the cardiovascular rehabilitation of patients with ischemic heart disease (13).

$\mathrm{CO}_{2}$ balneotherapy has effects on free radicals release and total antioxidant status in peripherical arterial occlusive disease. The free radical superoxide $\left(\mathrm{O}_{2}^{-}\right)$is produced as a by-product of oxidative phosphorylation in the mitochondria. $\mathrm{O}_{2}^{-}$is typically detoxified by superoxide dismutase (SOD), which makes them a more stable molecule of hydrogen peroxide $\left(\mathrm{H}_{2} \mathrm{O}_{2}\right)$ (14). Reactive oxygen species (ROS) are probably the most significant free radicals with substantial implications in cell damage and cells and body ageing (14). 
$\mathrm{CO}_{2}$ passes freely through membranes and has a wellknown vasodilatory effect. Both in vitro and in vivo studies have demonstrated a rightward shift of the oxygen-haemoglobin dissociation curve after administration of $\mathrm{CO}_{2}$, used to cure several diseases, such as peripheral arterial and venous disorders (e.g. claudication, and lower limb ulcer), heart diseases (e.g. hypertension, and heart failure) and immunological disorders (e.g. Raynaud's syndrome).

$\mathrm{CO}_{2}$ treatment is a non-invasive, highly efficient, lowcost treatment capable of easing the symptoms of arterial and venous diseases, possibly due to vasodilatation and reduction of oxidative stress. Consequently, an increase in sympathetic and parasympathetic activity can occur during $\mathrm{CO}_{2}$ treatment, resulting in unchanged heart rate, systolic and diastolic blood pressure. $\mathrm{CO}^{2}$ is not simply a vasodilator but can alter the function of the cardiovascular system, possibly by activating endogenous mechanisms leading to vasodilatation (15).

Hydrogen sulfide $\left(\mathbf{H}_{2} \mathrm{~S}\right)$ is both an exogenous therapeutic gas, found in mofettic combination with carbon dioxide, in sulphurous waters, with well known and appraised medicinal properties in balneotherapy, and also an endogenous gaseous signal molecule in organisms, which plays a vital role in various systems and diseases.

$\mathrm{H}_{2} \mathrm{~S}$ is mainly produced, in our organism, by three enzymes, including cystathionine $\gamma$-lyase, cystathionine $\beta$-synthase, and 3-mercaptopyruvate sulfur-transferase along with cysteine aminotransferase.

$\mathrm{H}_{2} \mathrm{~S}$ has relevance to neurologic disorders through antiapoptotic, antioxidative and anti-inflammatory effects. Recent basic medical and preclinical studies on neurologic diseases have demonstrated that the administration of $\mathrm{H}_{2} \mathrm{~S}$ at pharmacological or physiological levels attenuates brain injury.

$\mathrm{H}_{2} \mathrm{~S}$ was reported as a neuromodulator in the brain because of its essential role in facilitating long-term hippocampal potentiation at physiological concentration. However, the neuroprotective effect of $\mathrm{H}_{2} \mathrm{~S}$ is dependent on concentration. The only low dose of $\mathrm{H}_{2} \mathrm{~S}$, comparatively, can provide a beneficial effect.

$\mathrm{H}_{2} \mathrm{~S}$ is considered the third endogenous gaseous signal molecule in organisms, following nitric oxide (NO) and carbon monoxide $(\mathrm{CO})$.

Understanding of $\mathrm{H}_{2} \mathrm{~S}$ biological effect and its mechanism has been deepened, especially the physiopathologic significance of $\mathrm{H}_{2} \mathrm{~S}$ in various diseases such as neurological diseases, cardiovascular diseases, hematologic diseases, urological diseases, etc.

$\mathrm{H}_{2} \mathrm{~S}$ has many beneficial properties and may serve as a novel treatment in patients suffering from intestinal ischemia-reperfusion injury (16).
The protective effect of $\mathrm{H}_{2} \mathrm{~S}$ on neurons is expressed against oxidative stress by increasing the substrate for the production of the antioxidant $\mathrm{GSH}$, including the cystine/glutamate antiporter and the intracellular concentrations of Cys. It has been subsequently reported at the cellular level that $\mathrm{H}_{2} \mathrm{~S}$ also enhance the activity of the $\gamma$-glutamylcysteine synthase $(\gamma$-GCS). This ratelimiting enzyme regulates the generation of GSH. In addition, $\mathrm{H}_{2} \mathrm{~S}$ produced in mitochondria, the major organelle that releases reactive oxygen species (ROS), causing toxic effects and ultimately leading to cell death, also may directly suppress oxidative stress through scavenging ROS. These findings offer shreds of evidence for the influential anti-oxidative role of $\mathrm{H}_{2} \mathrm{~S}$.

$\mathrm{H} 2 \mathrm{~S}$ plays a protective role in inflammation by inhibiting lipopolysaccharide-stimulated tumour necrosis factor- $\alpha$ $(\mathrm{TNF}-\alpha)$, the proinflammatory cytokine interleukin-1 $\beta$ (IL- $\beta$ ) and NO release in astrocytes and microglial cells. Meanwhile, $\mathrm{H}_{2} \mathrm{~S}$ can increase the release of antiinflammatory cytokines, such as interleukin-4 (IL-4) or interleukin-10 (IL-10). As a result, $\mathrm{H}_{2} \mathrm{~S}$ may play an antiinflammatory role in the central nervous system (CNS).

$\mathrm{H}_{2} \mathrm{~S}$ may exert its anti-apoptotic role by inhibiting oxidative stress.

$\mathrm{H}_{2} \mathrm{~S}$ has vasculoprotective properties in endothelial cells and vascular smooth muscle cells, such as eliciting vasorelaxation and decreasing platelet aggregation.

$\mathrm{H}_{2} \mathrm{~S}$ possibly activates plasma membrane voltage-gated channels (L-type and T-type $\mathrm{Ca} 2+$ channels) and mobilized intracellular $\mathrm{Ca} 2+$ stores. In addition, endogenous $\mathrm{H}_{2} \mathrm{~S}$ was found to activate chloride $(\mathrm{Cl}-)$ channels and potassium $(\mathrm{K}+)$ channels, providing neuroprotective effects.

Neurodegenerative disease is an extensive term for a range of conditions that primarily affect the neurons in human brain, which are incurable and debilitating conditions that result in progressive degeneration and death of nerve cells.

A recent study has demonstrated that $\mathrm{H}_{2} \mathrm{~S}$ improved Hcyinduced cognitive dysfunction, which may play a beneficial role through inhibiting reactive aldehydes accumulation, preserving glutathione homeostasis, and upregulating aldehyde-dehydrogenase 2 activity and expression in the hippocampus of Hcy-exposed rats.

$\mathrm{H}_{2} \mathrm{~S}$ can reverse $\mathrm{A} \beta$-induced cognitive deficits via attenuating the production of $\mathrm{A} \beta$ and suppressing the down-regulation of CBS and 3-MST.

In addition, one study found that the progression of $\mathrm{AD}$ can be deterred through treatment with $\mathrm{H}_{2} \mathrm{~S}$ donors or spa-waters rich in $\mathrm{H}_{2} \mathrm{~S}$ content targeting multiple pathophysiological mechanisms appropriately.

In other $\mathrm{AD}$ models, the cytotoxic lipid oxidation product 4-hydroxynonenal was scavenged with $\mathrm{H}_{2} \mathrm{~S}$ therapy, which provides a novel hope against $\mathrm{AD}$ through the neuroprotection effects of $\mathrm{H}_{2} \mathrm{~S}$ (17). 


\section{Relevant data regarding medical gases used in rehabilitation medicine}

\section{Oxygen therapy}

Medical oxygen is considered a drug with beneficial effects at a safe dose and side effects at higher doses. Oxygen therapy is commonly used to treat or prevent hypoxemia. It is also indicated in patients with traumatic brain injury, haemorrhagic shock, resuscitation during cardiac arrest, and carbon monoxide poisoning. Generally, the required amount of supplemental O2 depends on the patient's condition. However, inappropriate prescription or administration of fraction of inspired oxygen may have negative consequences. Therefore, oxygen should be prescribed and delivered correctly to ensure safe and effective oxygen therapy. Based on current guidelines, the prescription should include the starting oxygen dose and oxygen delivery device, and most importantly, the oxygen saturation target range (18).

Oxygen supplementation (19) has been an ergogenic aid for patients with COPD. During exacerbations, patients with COPD may become significantly hypoxic (20).

Usually, many patients benefit from mechanical ventilation (VM) in acute mechanical failure, thus avoiding hypoxemia and hypercapnia. But, mechanical ventilation can cause lung damage, thus increasing the progression of the disease. Extracorporeal membrane oxygenation (ECMO) is, in this case, an alternative for rescuing patients with severe respiratory failure, for whom regular mechanical ventilation does not allow adequate and necessary gas exchange (21).

Oxygen can be administrated normobaric (normobaric oxygen therapy, NBOT) or under pressure (hyperbaric oxygen therapy, HBOT). Neuroprotective effects of oxygen therapy have been observed in various experimental models of brain injury and neurological diseases (22).

Patients with COPD in advanced stages have difficulty participating in outpatient pulmonary rehabilitation (PR) programs, requiring long-term oxygen therapy performed at home.

Their difficulty is determined by the severity of their disease, limitations involving mobility and transport, high costs and problems related to patient safety and individual needs (23).

Unsupervised home-based physical training (PT) is frequently used. On the contrary, supervised home-based PT is effective and safe in improving exercise capacity, dyspnea, fatigue, and patient health status (24).

Pulmonary rehabilitation (PR), including education, motivational support, and physical activity training, is the main non-pharmacological component of chronic obstructive pulmonary disease (COPD) treatment (25).
Recent clinical trials have investigated the role of supplemental oxygen during exercise training in rehabilitation programs. Patients with exercise-induced desaturation may not tolerate high-intensity exercise and may require reduced intensity training. It is postulated that oxygen may enhance the exercise training of patients with COPD (26). The benefit of pulmonary rehabilitation (PR) (27) in symptomatic chronic obstructive pulmonary disease (COPD) is well known (28). Recent studies about heated and humidified high flow oxygen therapy (HFOT) showed positive effects on breathing pattern and ventilatory efficiency during effort (29).

Home oxygen therapy (HOT), also named Long-term oxygen therapy (LTOT) (30), non-invasive ventilation (NIV) (31), and High-flow nasal cannula (HFNC) oxygen therapy (32), are included as new protocols of pulmonary rehabilitation of COPD, which may counteract the negative consequences and target modifiable risk factors of COPD patients for hospital readmission (33).

Long-term oxygen therapy is used by patients with chronic respiratory failure (CRF) due to advanced respiratory diseases. This therapy improves the prognosis of COPD. However, the therapeutic impact of long-term oxygen therapy does not fully reflect the "socialization" of patients, which is one of the final goals of "comprehensive pulmonary rehabilitation" (34).

Long-term oxygen therapy (35) is beneficial for patients with chronic resting hypoxemia. Ambulatory oxygen has also been used to treat patients who experience hypoxemia during routine daily activities but are normoxic at rest.

Studies performed on heated and humidified high flow gases delivered through a nasal cannula (HFNC) showed to (i) decrease respiratory rate, (ii) increase tidal volume, (iii) and reduced the work of breathing for patients with different forms of respiratory failure. The primary mechanism of action is the dead space washing-out, permitting a higher fraction of minute ventilation to take part in gas exchange. Moreover, HFNC can guarantee the desired inhaled oxygen fraction $(\mathrm{FiO} 2)$ even in patients with very high inspiratory flow rates (like during exercise) better than any other oxygen delivery system (36).

Multidisciplinary comprehensive respiratory rehabilitation combined with non-invasive positive pressure ventilation has high clinical values in the rehabilitation process of COPD patients, and can further relieve dyspnea and improve exercise endurance and living quality. Implementing appropriate and effective health education, rehabilitation guidance, medication guidance, nutritional guidance, and psycho-health guidance can further strengthen the recognition of patients on COPD and improve compliance (37). 
Hyperbaric oxygen therapy (HBOT) is considered a non-invasive method for treating patients sitting in a specially designed hyperbaric chamber, where they breathe $100 \%$ oxygen administered at a pressure higher than normal atmospheric pressure.

Hyperbaric oxygen therapy can help patients with hardto-heal wounds (after injuries, in the course of diabetic foot syndrome, and after radiotherapy), chronic osteomyelitis, bacterial tissue infection, carbon monoxide poisoning, and extensive burns (second-and third-degree burns covering over $20 \%$ of the body surface area). For therapeutic purposes, the pressure inside the chamber should exceed 1.4-atmosphere absolute (ATA) to increase the amount of oxygen supplied to cells in the body. During treatments, a pressure of 2.5 ATA is usually applied (38).

Hyperbaric oxygen therapy (HBOT) has neuroprotective effects and improves survival in animal models of middle cerebral artery occlusion (MCAO), especially in animals given more than 6 hours of $\mathrm{HBO}$ and given $\mathrm{HBO}$ at 2.0 ATA $(101.3 \mathrm{kPa})$ immediately after MCAO. In tPAinduced thrombolysis in vitro, HBO increased tPAinduced thrombolysis; and in rats subjected to thromboembolic MCAO, 5-minute HBO reduced infarct volume and brain edema (22).

Necrotizing soft tissue infections (NSTIs) are severe issues, sometimes deadly. NSTIs involve rapidly progressing soft-tissue inflammation with necrosis and can quickly cause multiple organ failure and death. Patients can become mortally infected in hours. Mortality is $25 \%-35 \%$, with survivors coping with amputations and prolonged rehabilitation. Septic shock accompanies death due to NSTI. Hyperbaric oxygen therapy (HBOT) may be used as adjunctive therapy of NSTI. Extensive database surveys indicate that HBOT improves the survival of these patients. HBOT is now used as part of the standard NSTI treatment (39).

The effects of HBOT on depression and anxiety are similar to psychotherapy. HBO improves significantly nerve function and activities of daily living in patients with incomplete Spinal Cord Injury (40), which either psychotherapy or routine rehabilitation therapy can not substitute. Hyperbaric oxygen (HBO), an essential treatment for nervous system injuries, can improve histanoxia, relieving edema.

HBO helps the recovery of neurological function in patients with cervical SCI. HBO down-regulates caspase3 expression and promotes neuron regeneration by improving blood supply and oxygen supply to the injured site, inhibiting, in this way apoptosis, after SCI.

HBO is also used to treat ischemic encephalopathy, cerebral apoplexy, traumatic brain injury, peripheral nerve injury (41), and multiple sclerosis (42).
Long-term effects of reduced vascularisation compromising the implantation site determine the significantly higher implant failure rates in maxillofacial patients undergoing radiotherapy. An extensive preclinical animal literature and many clinical reports suggest hyperbaric oxygen (HBO) therapy can improve tissue vascularity. To reducing the risk of implant failures in irradiated patients, preventive HBO therapy can be of help (43).

Many previous studies have reported the ability of HBO to improve implant osseointegration and prevent osteoradionecrosis (44)

Hyperbaric oxygen therapy is an attractive and logical option for treating $\mathrm{H} 2 \mathrm{~S}$ intoxication.

Effects of hyperbaric Oxygen Therapy on chronic cerebral stroke Patients were evaluated by Functional Magnetic Resonance Imaging (fMRI). Results indicate changes consistent with the clinical improvement in all chronic cerebral stroke Patients, suggesting a possible role of fMRI in revealing functional neuronal correlates of neuronal plasticity and HBOT-related neoangiogenesis. fMRI is a sensitive, non-invasive and reliable modality for monitoring neuronal functional changes before and after HBOT (45).

Aneurysmal subarachnoid hemorrhage is a cerebrovascular disease that seriously endangers public health. $85 \%$ of cases are spontaneous, and $3 \%$ to $5 \%$ of stroke. Although therapeutic approaches have progressed substantially, including surgery, interventional embolization, and intensive care, the death rate for this pathology is still very high.

Epidemiological studies show that the average mortality rate for aneurysmal subarachnoid hemorrhage is $27 \%$ to $44 \%$. On the other hand, many survivors also live with impaired cognitive and physical abilities, and more than $40 \%$ of patients with this condition cannot return to premorbid conditions. Therefore, improving the long-term prognosis in patients with aneurysmal subarachnoid hemorrhage remains critical. Although it is generally considered that early rehabilitation in the acute phase of the neurological lesion is effective, there is no consensus that this also happens in the case of aneurysmal subarachnoid hemorrhage. Early rehabilitation, in this case, can increase the risk of intracranial pressure and cerebral vasospasm, leading to secondary brain damage. Cerebral vasospasm and delayed cerebral ischemia represent leading causes of disability and death related to this disease. Hyperbaric oxygen therapy (HBOT) inhibits cerebral vasospasm by regulating the protein kinase pathway and inflammatory cytokine secretion. HBOT can also reduce the heart attack size and improve neurological function by inhibiting oxidative stress, reducing brain edema, increasing oxygen transport capacity, promoting the integrity of the blood-brain barrier, and increasing cerebral blood flow (46). 


\section{Helium}

Helium, a noble gas, has many applications due to its distinct physical and chemical characteristics: low density, low solubility, and high thermal conductivity. The abundance of medical studies related to helium mainly focuses on its possibility of adjunctive therapy in several respiratory diseases, such as exacerbation of COPD, asthma, ARDS, laryngotracheobronchitis and bronchiolitis. Helium gas has recently been shown to be biologically inert, beneficial in protecting the myocardium from ischemia by various mechanisms. Although neuroprotection of brain tissue was documented, how it does so has not yet been clarified. For laparoscopic abdominal procedures, surgeons are exploring helium instead of carbon dioxide to infuse the abdomen, owing to its superiority in preventing respiratory acidosis and because of carbon dioxide retention in patients with comorbid conditions. Helium inhalation enhances the vasodilator effect of inhaled nitric oxide on pulmonary vessels. Helium has been shown to protect myocardial tissue from ischemia through various mechanisms. Helium maintains intracellular acidosis during early reperfusion, which inhibits the formation of mitochondrial transition pores. The researchers found that reactive oxygen species (including nitric oxide), mitochondrial adenosine triphosphate-regulated potassium channels, and opioid receptors were responsible for the cardioprotective effects induced by helium preconditioning. Heinen et al. applied Heliox as a preconditioning method in a model of rat cardiac ischemia and reperfusion. The results showed that the cardio-protective effects of Heliox preconditioning in young rats were due to two additional mechanisms: mitochondrial decoupling and activation of the $\mathrm{Ca} 2+$ sensitive potassium channel. Helium protects the healthy myocardium against ischemia and reperfusion injury through early and late preconditioning (EPC, LPC) and postconditioning (PostC). In 2012, Weber et al. found that triple intervention of EPC, LPC and PostC significantly reduced the size of the infarction in spontaneously hypertensive rats.

The clinical use of helium-oxygen mixtures (Heliox) has been supported for several conditions, such as upper airway obstruction, croup, acute asthma, and postextubation stridor. Heliox has been used in patients with chronic obstructive pulmonary disease (COPD), assuming that this mixture of gases, due to its low density, can reduce lung resistance. Therefore, it is worth briefly reviewing the physical properties of Heliox and their impact on the dynamics of the respiratory system. Due to its low solubility, helium crosses the alveolarcapillary membrane very slowly, although its diffusibility is higher than that of oxygen, carbon dioxide and even nitrogen. Two electrons fill the unique orbital of helium, so helium does not form compounds. It is considered to be metabolically inert and appears as a colorless, odorless and tasteless gas. Unlike xenon, it lacks anesthetic properties. As a therapeutic gas, helium is used to replace nitrogen as an oxygen-carrying gas. The percentage of oxygen in Heliox must be at least $20 \%$ to prevent hypoxia and not more than $40 \%$.

Beyond this value, Heliox is unlikely to exert any relevant clinical effect. The density of a helium-oxygen mixture can be obtained as a weighted average density of oxygen and helium (47).

High-intensity training is promising as a time-efficient exercise strategy in cardiovascular rehabilitation. Agreement on a more uniform exercise protocol is essential to further investigate the optimal exercise mode for cerebrovascular rehabilitation (48).

Heliox and oxygen breathing reduce dyspnea during exercise. Regarding dyspnea, an interesting observation, in this case, is that Heliox and oxygen acted differently on the reduction of exertional dyspnea. Indeed, heliox supplementation as an ergogenic strategy is based on the idea that a lighter gas mixture could be moved more easily to and from the pulmonary airways than atmospheric air; Heliox reduces turbulent airway resistance and inspiratory and expiratory pressures during exercise, compared to breathing air from the room. Respiration with Heliox and oxygen: central and local hemodynamic responses during exercise The beneficial effects of Heliox and supplemental oxygen on metabolic muscle function and contractions of the respiratory and locomotor muscles would delay the onset of lactic acidosis, accompanied by improvements in dyspnoea, exercise and discomfort. tolerance in patients with COPD. In patients with COPD, the ergogenic effect of Heliox or oxygen respiration may be related to improved ventilatory parameters that contribute to decreased dyspnea and increased oxygen delivery to the respiratory and locomotor muscles. Rehabilitation training exercises using Heliox or oxygen supplementation should focus on increasing the intensity of training to achieve more significant physiological training benefits (49).

Nitric oxide (NO) is considered an essential signal molecule involved in physiological and pathological processes. Nitric oxide is a neurotransmitter in the central nervous system. NO regulates cerebral blood flow, neurogenesis, and synaptic plasticity (50). Nitric oxide is produced in the airways of asthmatic subjects and is linked to $\mathrm{T} 2$ inflammation (51).

The medical significance of NO inhaled as a selective pulmonary vasodilator is based on its characteristic of being administered as a direct gas in the pulmonary circulation, without systemic side effects. Nitric oxide activates guanylate cyclase and converts it to cyclic guanine monophosphate (cGMP). The presence of cGMP at the smooth muscle causes relaxation. 


\section{Molecular hydrogen $\mathrm{H} 2$}

$\mathrm{H} 2$ is the lightest gas, commonly used as a component of the exotic mixture of respiratory gases, namely hydreliox $(49 \% \mathrm{H} 2,50 \%$ helium and $1 \% \mathrm{O} 2)$ for deep-water divers to prevent decompression sickness. The therapeutic application of $\mathrm{H} 2$ as a free radical catalyst began in the 1970s. The antioxidant benefits of H2 gas included: 1) high biomembrane penetration and intracellular diffusion capacity that allow it to reach subcellular compartments such as mitochondria; and 2) the selective elimination of the harmful hydroxyl radical while retaining other important reactive oxygen and nitrogen species for normal signaling regulation. It is superior to antioxidant supplements with intense reductive activity, such as vitamin $\mathrm{E}$ or vitamin $\mathrm{C}$, to avoid the increased risk of mortality.

$\mathrm{H} 2$ is now considered a signaling gas molecule with physiological functions similar to nitric oxide (NO), carbon monoxide (CO) and H2S. Indeed, H2 does not have cytotoxicity even at a high concentration, ensuring the safety privilege compared to other gases.

Three forms of molecular hydrogen administration, namely inhalation with $1-4 \%$ hydrogen gas, hydrogenrich intraperitoneal saline injection / intravenous infusion and oral hydrogen saturated water intake, have been commonly used in hydrogen medical research. H2 concentrations in tissues depend on the $\mathrm{H} 2$ concentration administered, and the specific $\mathrm{H} 2$ absorption of the tissue is related to the difference in route of administration, indicating the importance of choosing the most efficient delivery route and the dose of hydrogen for each disease or tissue. The therapeutic effect of molecular hydrogen $\mathrm{H} 2$ has been demonstrated in the central nervous system, cardiovascular system, lung, kidneys, liver, pancreas, skin, eyes, bones and reproductive system, which have the underlying pathological conditions of ischemicreperfusion injury (including organ transplantation):) and predominant diseases mediated by oxidative stress. Although the basic mechanisms were initially proposed as selective extinctions of the hydroxyl radical and peroxynitrite, regulating the molecular hydrogen signaling pathway by modulating different expressions/activities of molecules, gene expression and microRNA may also explain the final effects of the lesion. anti-reperfusion, anti-inflammatory, antiapoptosis, anti-metabolic, anti-allergic, anti-radiation, anti-dementia, and anti-aging disorders. Small cohort studies or case reports have revealed the safety or some promising benefits of therapeutic hydrogen in a wide range of diseases and conditions, such as post-cardiac syndrome, Parkinson's disease, acute cerebral ischemia, metabolic syndrome, rheumatoid arthritis. Hemodialysis and post-psoriasis. Several large-scale prospective clinical trials on Parkinson's disease, acute post-cardiac arrest syndrome and myocardial infarction, as well as cerebral infarction, are ongoing (52).

Interestingly, $\mathrm{H} 2$ appears to be an effective ROS scavenging drug. Thus, the inhalation of gaseous $\mathrm{H} 2$ may be a viable treatment option for patients with asthma and/or COPD (53).

\section{Ozone therapy}

Ozone $(\mathrm{O} 3)$ is an inorganic molecule with allotropic properties consisting of three oxygen atoms with a cyclic structure. $\mathrm{O} 3$ is present in nature in the stratosphere. However, it can also be produced artificially by subjecting diatomic oxygen $(\mathrm{O} 2)$ to a high-voltage electrical discharge and appears colorless in gaseous form with a characteristic odor. In recent years, regarded in the use of oxygen-ozone (O2O3) therapy has increased considerably in rehabilitation. Despite its widespread use in standard clinical practice, the biochemical effects of $\mathrm{O} 2 \mathrm{O} 3$ are still far from being understood. However, its chemical properties seem to play a key role in exerting its positive impact on various pathological conditions. Indeed, the effectiveness of $\mathrm{O} 2 \mathrm{O} 3$ therapy may be due in part to the moderate oxidative stress produced by $\mathrm{O} 3$ interactions with biological components. $\mathrm{O} 2 \mathrm{O} 3$ therapy is widely used as an adjunctive therapeutic option in several pathological conditions characterized by chronic inflammatory processes and immune overactivation, and most musculoskeletal disorders share these pathophysiological processes. $\mathrm{O} 2 \mathrm{O} 3$ could be used with an indirect and minimally invasive approach, targeting the paravertebral muscles corresponding to the herniated disc metamer. $\mathrm{O} 2 \mathrm{O} 3$ therapy has been proposed for several years as an effective treatment in patients with LBP due to intervertebral disc herniation. LBP is the most ordinary musculoskeletal disorder in the general population and almost everyone will experience LBP at least once in their lifetime (54). Another site of interest for $\mathrm{O} 2 \mathrm{O} 3$ therapy is the knee joint (55).

Intraarticular injections of $\mathrm{O} 2 \mathrm{O} 3$ for osteoarthritis of the knee relieve pain, reduce discharge, improve mobility of the knee joints and have good efficacy, especially during the exacerbation of stage I to II pain on the Kellgren Lawrence (KL) scale. Intra-articular $\mathrm{O} 2 \mathrm{O} 3$ injections are safe and encourage short- and medium-term effects on pain control and functional recovery in patients with KOA (56). O2O3 injections inhibit proinflammatory cytokines, such as prostaglandin E2, favoring antiinflammatory cytokines such as interleukin 10 , transforming growth factor $\mathrm{b}$, and interleukin 4; antioxidant enzymes; and angiogenesis. These components cooperate to repair the joint by stimulating fibroblasts, chondrocytes and stem cells (57).

Xenon has neuroprotective properties, and treatment with xenon showed protection of cell viability (58) 
Argon appertains to the group of noble gases, which are considered chemically inert. Amazingly, some of these gases have biological properties, and, in recent decades, more and more reports have shown neuroprotective and organ protective effects. Recent studies predominantly use in vivo or in vitro models for ischemic pathologies to investigate the impact of argon treatment. Promising data have been published on pathologies such as cerebral ischemia, traumatic brain injury and hypoxic-ischemic encephalopathy. However, the models applied and the administration of therapeutic gas vary. In general, the most promising therapies - especially neuroprotective identified by preclinical studies have failed to demonstrate efficacy in clinical trials due to heterogeneous experimental settings, inadequate sample size, timing and inappropriate doses (58).

We know very little about the mechanism of action of argon. At atmospheric pressure, argon did not cause macrophage intracellular acidosis induced by other benzodiazepine-sensitive GABAA receptor agonists. Thus, two distinct, action-independent methods are designed, depending on the environmental pressure and the measured response. Similar to xenon, which inhibits NMDA receptors, this type of receptor was investigated during argon treatment (59).

Volatile Anesthetics routinely used in clinics. Hence, the volatile anesthetics such as isoflurane and sevoflurane, are considered as low-risk-bearing gaseous agents. Application of commonly used volatile anesthetics after brain ischemia onset provides neuroprotection in experimental stroke research. Post-conditioning with $3.0 \%$ isoflurane provided better neuroprotection than $1.5 \%$ and $4.5 \%$ isoflurane. Effects of isoflurane postconditioning were mediated by activation of activin $\mathrm{A} / \mathrm{Smad} 2 / 3$ and activin A/extracellular signal-regulated kinase (ERK) 1/2 signaling pathway. However, in another study, $1.5 \%$ isoflurane post-conditioning was showed to be more effective than $3.0 \%$, and $4 \%$ isoflurane in reducing infarct volume and improving neurological deficits, which were associated with upregulated expression of transforming growth factor-beta 1 (TGF- $\beta 1$ ) and down-regulated phosphorylated c-Jun Nterminal kinase ( $\mathrm{p}-\mathrm{JNK}$ ) expression.

Rabbit post-conditioned with isoflurane tolerates more clots than control animals in the dose-response study, and isoflurane post-conditioning reduced infarct volume and improved neurological deficit scores in animals received an intra-carotid injection of $5 \mathrm{mg}$ clots. Although preclinical studies provide strong evidences that isoflurane induces neuroprotection, clinical results, especially long-term neurological outcomes, are disappointing.

Translation of positive animal findings to patients has been hampered by the associated clinical comorbidity and concurrent medication of patients (22).

\section{DISCUSSION}

Interesting, regarding mud: it is a heterogeneous physicochemical system which comprises a liquid phase containing water-soluble mineral salts and water, a solid phase containing minerals and organic substances, but also a gaseous phase containing hydrogen sulfide, carbon dioxide, hydrocarbons, etc., some therapeutical mechanisms of mud relying on this gas content.

Responses of cerebral blood flow to exercise are enhanced by an associate of the rehabilitation program. The increased ability of the brain to respond to physiological and regulatory stimuli is important for maintaining a healthy brain throughout life. Although several cross-sectional studies indicate an increase in general cerebrovascular function in people trained in exercise, a more recent study showed that dynamic cerebrovascular self-regulation is slightly attenuated in athletes after 6 weeks of high-intensity training (60).

$\mathrm{CO} 2$ balneotherapy has long been applied clinically to improve cardiovascular symptoms in European countries. Instead, Carbon dioxide triggers peripheral vasodilatation in blood vessels following inhalation or immersion. Intracorporal variations in $\mathrm{CO} 2$ concentrations affect both blood flow, tissue perfusion, and the capacity of hemoglobin to release oxygen. $\mathrm{CO} 2$ bathing has an old history and is thought to be effective in the treatment of a peripheral vascular disorder, too (61).

Corollary, some evidence supports the idea that $\mathrm{CO} 2$ baths might represent an efficient therapeutic means in the rehabilitation of coronary heart disease, myocardial infarction, and stroke, and in the treatment of chronic venous insufficiency, certain inflammatory diseases, and functional disturbances. $\mathrm{CO} 2$ acts in different manners: increases skin blood flow during $\mathrm{CO} 2$-water immersion facilitates heat transfer from the body to the surrounding water, and also $\mathrm{CO} 2$ inhibits the activity of cold receptors and facilitates the activity of warm-sensitive receptors in the skin, affecting the firing rates of preoptic thermosensitive neurons; these activated in response to peripheral or central temperature stimuli.

Given that ROS formation is common in asthma and COPD, the use of free radical scavengers, such as hydrogen sulfide $\left(\mathrm{H}_{2} \mathrm{~S}\right)$, may be an effective adjuvant treatment. $\mathrm{H}_{2} \mathrm{~S}$ can relax smooth muscle in the intrapulmonary airways by inhibiting the intracellular release of $\mathrm{Ca}^{2+}$. On the other hand, $\mathrm{H}_{2} \mathrm{~S}$ has antioxidant and anti-inflammatory properties. For example, exogenous $\mathrm{H}_{2} \mathrm{~S}$ can reverse ovalbumin-induced asthma.

The concentration of $\mathrm{H}_{2} \mathrm{~S}$ is increased in sputum supernatants and the serum of asthma patients, while lung function is inversely correlated with sputum $\mathrm{H}_{2} \mathrm{~S}$ levels. Therefore, H2S may serve as a molecular marker in patients diagnosed with asthma (53). Both discussed gases, $\mathrm{CO} 2$ and $\mathrm{H} 2 \mathrm{~S}$ can be present in the gaseous phase of peloids and the moffetic gas combination. 
Oxygen therapy, used very often in the clinic, should be carefully administered according to the doctor's prescription. Molecular oxygen $(\mathrm{O} 2)$ is a vital element for the body's survival and plays a significant role in diverse bio-physiological processes. Although normobaric hyperoxia may increase arterial oxygen content, it also causes vasoconstriction and therefore reduces the delivery of $\mathrm{O} 2$ to various vascular regions, including the heart, skeletal muscle, and brain. Thus, there is a seemingly paradoxical situation in which oxygen administration can cause an increased risk of hypoxic stress in the tissues. However, with varying degrees of efficacy, and not without consequences, supplemental oxygen is used clinically to correct tissue hypoxia (e.g., cerebral ischemia, traumatic brain injury, carbon monoxide poisoning, etc.) and chronic hypoxemia (e.g. obstructive pulmonary disease (COPD) (62), etc.) and to help heal wounds, necrosis or reperfusion lesions (e.g. compromised grafts).

Hyperoxia has also been used freely by athletes, considering that it offers benefits that improve performance; such benefits extend to hypoxemic patients both at rest and during rehabilitation (63).

Home oxygen therapy (HOT), also known as long-term oxygen therapy, is prescribed to patients with chronic respiratory failure (CRF) due to advanced respiratory diseases, as it has been shown to improve the prognosis of patients with chronic obstructive pulmonary disease (COPD). However, the therapeutic impact of HOT does not fully reflect the "socialization" of patients, which is one of the final goals of "comprehensive pulmonary rehabilitation" (22).

The latest inhaler gas to receive research attention for a possible therapeutic application is carbon monoxide (CO). $\mathrm{CO}$ is regarded as a toxic element of cigarette smoke and a consistent component of air pollution worldwide. Inhaled $\mathrm{CO}$ binds to hemoglobin from our blood to form carboxyhemoglobin, which disrupts regular oxygen transport. Carbon monoxide is odorless and colorless. Symptoms of overexposure include headache, dizziness, heart palpitations, weakness, confusion, nausea, seizures, unconsciousness, and eventually death. However, scientific investigations have found that at a concentration of 250-1,000 ppm, CO is an essential vascular paracrine factor. Recently, experimental studies have shown the benefits of inhaled $\mathrm{CO}$ on the progress of different lung disorders, using in vivo models of induced ventilator lung damage, aspiration, hyperoxia, and ischemia-reperfusion. The physiological basis for benefiting from inhaled $\mathrm{CO}$ is the recent discovery of the stress protein heme oxygenase- 1 , which degrades heme into biliverdin-IX alpha, $\mathrm{CO}$, and iron. $\mathrm{CO}$ can provide cytoprotective and tissue-protective effects at low concentrations that involve inhibition of inflammatory, proliferative and apoptotic signaling.
The protection of the lungs by heme oxygenase- 1 has been demonstrated in vitro and in vivo in several sepsis and acute lung injury models. Published studies have also examined the protective effects of pharmacological or inhaled CO therapy on animal models of acute lung injury and sepsis. $\mathrm{CO}$ has demonstrated therapeutic potential in models of oxidative and acid-induced lung lesions, ventilator-induced lung injection, endotoxin challenge and cecal ligation, and puncture-induced sepsis. As inhaled CO is only investigative at this time, there are no published guidelines for indications. Any use of inhaled CO must be carried out with the approval of an institutional review committee governing the research on human subjects (3).

\section{Conclusions}

Therapeutic gases are not fully understood and used adequately for sanogenic or medical purposes. More research is needed to fully understand the mechanisms and importance of therapeutic gases. The link between balneotherapy and medical rehabilitation regarding the usage of therapeutical gases emphasizes the unity for this medical specialty.

\section{Funding}

This research did not receive any specific grant from funding public, commercial, or not-for-profit agencies.

\section{Acknowledgments}

This study did not require written consent from patients. All authors have equally contributed to this article.

\section{Declaration of interests}

Authors declare no competing interests. Approval of the Ethics Commission of „Bagdasar-Arseni” Hospital in Bucharest (N.O. 9910/17.03.2021) was obtained.

\section{References:}

1. Verhagen AP, Bierma-Zeinstra SMA, Boers M, Cardoso JR, Lambeck J, de Bie R, de Vet HCW - Balneotherapy (or spa therapy) for rheumatoid arthritis (Review). 2015;

2. Nasermoaddeli A, Kagamimori S. Balneotherapy in medicine: A review. Environ Health Prev Med. 2005;10(4):171-9.

3. Gentile MA. Inhaled medical gases: More to breathe than oxygen. Respir Care. 2011;56(9):1341-57.

4. Liu W, Khatibi N, Sridharan A, Zhang JH. Application of medical gases in the field of neurobiology. Med Gas Res [Internet]. 2011;1(1):13. Available from: http://www.medicalgasresearch.com/content/1/1/13

5. Kelly LP, Devasahayam AJ, Chaves AR, Wallack EM, McCarthy J, Basset FA, et al. Intensifying functional task practice to meet aerobic training guidelines in stroke survivors. Front Physiol. 2017;8(OCT):1-9.

6. Onose G, Popescu N, Munteanu C, Ciobanu V, Sporea C, Mirea MD, et al. Mobile mechatronic/robotic orthotic devices to assist-rehabilitate neuromotor impairments in the upper limb: A systematic and synthetic review. Front Neurosci. 2018;12(SEP).

7. Munteanu C, Rotariu M, Dogaru G, Ionescu VE, Vlad C, Onose G. Mud therapy and rehabilitation - scientific relevance in the last six years $(2015$ - 2020) Systematic literature review and meta-analysis based on the PRISMA 
paradigm. 2021;12(1):1-15.

8. Munteanu C, Păun D-L, Șuță A-M, Florescu SA, Onose G. Diabetes mellitus and COVID-19 in the post-acute phase patients - possible links with physical and rehabilitation medicine and balneotherapy. Balneo Res J. 2020;11(Vol.11, 3):350-67.

9. Munteaanu C, Munteanu D. Thalassotherapy today. Vol. 10, Balneo Research Journal. 2019. p. 440-4.

10. Bulboaca AE, Boarescu PM, Porfire AS, Dogaru G, Barbalata C, Valeanu $M$, et al. The effect of nanoepigallocatechin-gallate on oxidative stress and matrix metalloproteinases in experimental diabetes mellitus. Antioxidants. 2020;9(2).

11. Dogaru G, Marieta M, Bulboacă A, Ciumărnean L, Stănescu I. The effect of carbonated mineral water and mofette treatment in Baile Tusnad after ischemic stroke a case report. Balneo Res J. 2018;9(1):11-4.

12. Dogaru G, Bulboaca A, Boarescu PM, Ciumarnean L, Rus V, Sitar-Taut AV, et al. The effect of mofettes on oxidative stress/antioxidant balance in experimental myocardial ischemia. In Vivo (Brooklyn). 2019;33(6):1911-20.

13. Suceveanu M, Suceveanu P, Pop D, Sitar Taut A, ZDRENGHEA D, HÂNCU N. Role of Mofette Therapy in Cardiovascular Rehabilitation - the Covasna Model -. Balneo Res J. 2015;6(2):69-74.

14. Munteanu C D, Munteanu. Healthy aging. Balneo Res J. 2013;4(3):121-32.

15. Kreska Z, Németh B, Kiss I, Péter I, Ajtay Z, Hejjel L. Transcutaneous carbon dioxide treatment affects heart rate variability - A pilot study. In Vivo (Brooklyn). 2018;32(5):1259-64.

16. Jensen, Amanda R.; Drucker, Natalie A.; Winkel J an PF. The Route and Timing of Hydrogen Sulfide Therapy Critically Impacts Intestinal Recovery Following Ischemia and Reperfusion Injury. Physiol Behav. 2018;176(5):139-48.

17. Zhang JY, DIng YP, Wang Z, Kong Y, Gao R, Chen G. Hydrogen sulfide therapy in brain diseases: From bench to bedside. Med Gas Res. 2017;7(2):113-9.

18. Al-Otaibi HM. Current practice of prescription and administration of oxygen therapy: An observational study at a single teaching hospital. J Taibah Univ Med Sci [Internet]. 2019;14(4):357-62. Available from: https://doi.org/10.1016/j.jtumed.2019.05.004

19. Leung RWM, Alison JA, Jenkins SC, Holland AE, Hill $\mathrm{K}$, Morris NR, et al. Use of supplemental oxygen during exercise testing and training for people with chronic obstructive pulmonary disease: a survey of Australian pulmonary rehabilitation programs. Brazilian J Phys Ther [Internet]. 2021;25(1):97-102. Available from: https://doi.org/10.1016/j.bjpt.2020.04.003

20. Halpin DMG, Miravitlles M, Metzdorf N, Celli B. Impact and prevention of severe exacerbations of COPD: A review of the evidence. Int J COPD. 2017;12:2891-908.

21. Zhang Z, Gu WJ, Chen K, Ni H. Mechanical Ventilation during Extracorporeal Membrane Oxygenation in Patients with Acute Severe Respiratory Failure. Can Respir J. 2017;2017.

22. Huang JL, Zhao BL, Manaenko A, Liu F, Sun XJ, Hu Q. Medical gases for stroke therapy: Summary of progress
2015-2016. Med Gas Res. 2017;7(2):107-12.

23. Rondanelli M, Faliva MA, Peroni G, Infantino V, Gasparri C, Iannello G, et al. Food pyramid for subjects with chronic obstructive pulmonary diseases. Int J COPD. 2020;15:1435-48.

24. Kovelis D, Gomes ARS, Mazzarin C, Biazim SK, Pitta F, Valderramas S. Effectiveness and Safety of Supervised Home-Based Physical Training in Patients With COPD on Long-term Home Oxygen Therapy: A Randomized Trial. Chest [Internet]. 2020;158(3):965-72. Available from: https://doi.org/10.1016/j.chest.2020.02.063

25. Gephine S, Rouzic O Le, Machuron F, Wallaert B, Chenivesse C, Saey D, et al. Long-term effectiveness of a home-based pulmonary rehabilitation in older people with chronic obstructive pulmonary disease: A retrospective study. Int J COPD. 2020;15:2505-14.

26. Liu Y, Gong F. Determination of whether supplemental oxygen therapy is beneficial during exercise training in patients with COPD: A systematic review and meta-analysis. Exp Ther Med. 2019;4081-9.

27. Miki K, Maekura R, Kitada S, Miki M, Yoshimura K, Yamamoto $\mathrm{H}$, et al. Pulmonary rehabilitation for COPD improves exercise time rather than exercise tolerance: effects and mechanisms. Int J COPD. 2017;12:1061-70.

28. Ou YE, Lin ZM, Hua DM, Jiang Y, Huo YT, Luo Q, et al. Evaluation of carbon dioxide rebreathing during exercise assisted by noninvasive ventilation with plateau exhalation valve. Int J COPD. 2017;12:291-8.

29. Vitacca M, Pietta I, Lazzeri M, Paneroni M. Effect of high-flow nasal therapy during exercise training in COPD patients with chronic respiratory failure: Study protocol for a randomised controlled trial. Trials. 2019;20(1):1-8.

30. Coquart JB, Le Rouzic O, Racil G, Wallaert B, Grosbois JM. Real-life feasibility and effectiveness of home-based pulmonary rehabilitation in chronic obstructive pulmonary disease requiring medical equipment. Int $\mathrm{J}$ COPD. 2017;12:3549-56.

31. Polastri M, Schifino G, Tonveronachi E, Tavalazzi F. Respiratory Treatment in a Patient with Nemaline Myopathy. Clin Pract. 2019;9(4):123-6.

32. Torres A, Crisafulli E, Barbeta E, Ielpo A. Management of severe acute exacerbations of COPD: an updated narrative review. Multidiscip Respir Med. 2018;13:1-15.

33. Fu C, Liu X, Zhu Q, Wu X, Hao S, Xie L, et al. Efficiency of High-Flow Nasal Cannula on Pulmonary Rehabilitation in COPD Patients: A Meta-Analysis. Biomed Res Int. 2020;2020.

34. Wada H, Fukumoto S, Tamamura K, Muto G, Ueki J, Tanigawa T. Toward the harmonization of work with treatment and prevention for patients with chronic respiratory failure. Ind Health. 2019;57(1):84-9.

35. Vitacca M, Kaymaz D, Lanini B, Vagheggini G, Ergün P, Gigliotti F, et al. Non-invasive ventilation during cycle exercise training in patients with chronic respiratory failure on long-term ventilatory support: A randomized controlled trial. Respirology. 2018;23(2):182-9.

36. Cirio S, Piran M, Vitacca M, Piaggi G, Ceriana P, Prazzoli M, et al. Effects of heated and humidified high flow gases during high-intensity constant-load exercise on severe COPD patients with ventilatory limitation. Respir Med [Internet]. 2016;118:128-32. Available from: 
http://dx.doi.org/10.1016/j.rmed.2016.08.004

37. Cui L, Liu H, Sun L. Multidisciplinary respiratory rehabilitation in combination with non-invasive positive pressure ventilation in the treatment of elderly patients with severe chronic obstructive pulmonary disease. Pakistan J Med Sci. 2019;35(2):500-5.

38. Skiba M, Rękas-Dudziak A, Bekała A, Płotek W. Late application of hyperbaric oxygen therapy during the rehabilitation of a patient with severe cognitive impairment after a traumatic brain injury. Clin Case Reports. 2021;9(2):960-5.

39. Polzik P, Johansson PI, Hyldegaard O. How biomarkers reflect the prognosis and treatment of necrotising soft tissue infections and the effects of hyperbaric oxygen therapy: The protocol of the prospective cohort PROTREAT study conducted at a tertiary hospital in Copenhagen, Denmark. BMJ Open. 2017;7(10):1-7.

40. Munteanu C. Cell biology considerations in Spinal Cord Injury - Review. Balneo Res J. 2017;8(3):136-51.

41. Feng JJ, Li YH. Effects of hyperbaric oxygen therapy on depression and anxiety in the patients with incomplete spinal cord injury (a STROBE-compliant article). Med (United States). 2017;96(29).

42. Amatya B, Khan F, Galea M. Rehabilitation for people with multiple sclerosis: An overview of Cochrane Reviews. Cochrane Database Syst Rev. 2019;2019(1).

43. Shah DN, Chauhan CJ, Solanki JS. Effectiveness of hyperbaric oxygen therapy in irradiated maxillofacial dental implant patients: A systematic review with metaanalysis. J Indian Prosthodont Soc. 2017;17(2):109-27.

44. Awadalkreem F, Khalifa N, Ahmad AG, Suliman AM, Osman M. Rehabilitation of an irradiated marginal mandibulectomy patient using immediately loaded basal implant-supported fixed prostheses and hyperbaric oxygen therapy: A 2-year follow-up. Int J Surg Case Rep [Internet]. 2020;71:297-302. Available from: https://doi.org/10.1016/j.ijscr.2020.05.018

45. Cevolani D, Di Donato F, Santarella L, Bertossi S, Cellerini M. Functional MRI (fMRI) evaluation of hyperbaric oxygen therapy (HBOT) efficacy in chronic cerebral stroke: A small retrospective consecutive case series. Int J Environ Res Public Health. 2021;18(1):1-14.

46. Wang Y, Gao Y, Lu M, Liu Y. Long-term functional prognosis of patients with aneurysmal subarachnoid hemorrhage treated with rehabilitation combined with hyperbaric oxygen: Case-series study. Med (United States). 2020;99(3):1-5.

47. Pecchiari M. Effects of heliox in stable COPD patients at rest and during exercise. Pulm Med. 2012;2012.

48. Kolmos M, Krawcyk RS, Kruuse C. Effect of highintensity training on endothelial function in patients with cardiovascular and cerebrovascular disease: A systematic review. SAGE Open Med. 2016;4:205031211668225.

49. Louvaris Z, Vogiatzis I. Contrasting the physiological effects of heliox and oxygen during exercise in a patient with advanced COPD. Breathe. 2019;15(3):250-7.

50. Cichoń N, Czarny P, Bijak M, Miller E, Śliwiński T, Szemraj J, et al. Benign Effect of Extremely LowFrequency Electromagnetic Field on Brain Plasticity
Assessed by Nitric Oxide Metabolism during Poststroke Rehabilitation. Oxid Med Cell Longev. 2017;2017.

51. Pignatti $P$, Visca D, Loukides S, Märtson AG, Alffenaar JWC, Migliori GB, et al. A snapshot of exhaled nitric oxide and asthma characteristics: experience from high to low income countries. Pulmonology [Internet]. 2020; Available from: https://doi.org/10.1016/j.pulmoe.2020.10.016

52. Huang L. Molecular hydrogen: A therapeutic antioxidant and beyond. Med Gas Res. 2016;6(4):219-22.

53. Zuo L, Lucas K, Fortuna CA, Chuang CC, Best TM. Molecular regulation of toll-like receptors in asthma and COPD. Front Physiol. 2015;6(NOV):1-10.

54. Bellomo RG, Paolucci T, Giannandrea N, Pezzi L, Saggini R. Ozone therapy and aquatic rehabilitation exercises to overcome the lumbar pain caused by facet joint syndrome - case report. Int Med Case Rep J. 2020;13:171-6.

55. de Sire A, Agostini F, Lippi L, Mangone M, Marchese S, Cisari $\mathrm{C}$, et al. Oxygen-ozone therapy in the rehabilitation field: State of the art on mechanisms of action, safety and effectiveness in patients with musculoskeletal disorders. Biomolecules. 2021;11(3):1-17.

56. Gaballa NM, Mohammed YA, Kamel LM, Mahgoub HM. Therapeutic efficacy of intra-articular injection of platelet-rich plasma and ozone therapy in patients with primary knee osteoarthritis. Egypt Rheumatol [Internet]. 2019;41(3):183-7. Available from: https://doi.org/10.1016/j.ejr.2018.07.005

57. Paolucci T, Agostini F, Bernetti A, Paoloni M, Mangone $\mathrm{M}$, Santilli $\mathrm{V}$, et al. Integration of focal vibration and intra-articular oxygen-ozone therapy in rehabilitation of painful knee osteoarthritis. J Int Med Res. 2021;49(2).

58. Höllig A, Schug A, Fahlenkamp A V., Rossaint R, Coburn M, Brücken A, et al. Argon: Systematic review on neuro- and organoprotective properties of an "Inert" gas. Int J Mol Sci. 2014;15(10):18175-96.

59. Berganza CJ, Zhang JH. The role of helium gas in medicine. Med Gas Res [Internet]. 2013;3(1):18. Available from: Medical Gas Research

60. Smith KJ, Moreno-Suarez I, Scheer A, Dembo L, Naylor LH, Maiorana AJ, et al. Cerebral blood flow responses to exercise are enhanced in left ventricular assist device patients after an exercise rehabilitation program. J Appl Physiol. 2020;128(1):108-16.

61. Dogliotti G, Galliera E, Iorio E, De Bernardi Di Valserra M, Solimene U, Corsi MM, et al. CO 2 balneotherapy: effect on free radicals release and total antioxidant status in peripherical arterial occlusive disease. :1-12.

62. Cousins JL, Wood-Baker R, Wark PAB, Yang IA, Gibson PG, Hutchinson A, et al. Management of acute COPD exacerbations in Australia: do we follow the guidelines? ERJ Open Res [Internet]. 2020;6(2):00270-2019. Available http://dx.doi.org/10.1183/23120541.00270-2019

63. Brugniaux JV, Coombs GB, Barak OF, Dujic Z, Sekhon MS, Ainslie PN. Highs and lows of hyperoxia: Physiological, performance, and clinical aspects. Am J Physiol - Regul Integr Comp Physiol. 2018;315(1):R1R27. 